\title{
Minimizing Polynomials via Sum of Squares over the Gradient Ideal
}

\author{
James W. Demmel, Jiawang Nie ${ }^{\dagger}$ and Bernd Sturmfels ${ }^{\ddagger}$
}

\begin{abstract}
A method is proposed for finding the global minimum of a multivariate polynomial via sum of squares (SOS) relaxation over its gradient variety. That variety consists of all points where the gradient is zero and it need not be finite. A polynomial which is nonnegative on its gradient variety is shown to be SOS modulo its gradient ideal, provided the gradient ideal is radical or the polynomial is strictly positive on the gradient variety. This opens up the possibility of solving previously intractable polynomial optimization problems. The related problem of constrained minimization is also considered, and numerical examples are discussed. Experiments show that our method using the gradient variety outperforms prior SOS methods.
\end{abstract}

Key Words: Polynomials, global optimization, Sum of Squares (SOS), SemidefiniteProgramming (SDP), radical ideal, variety, gradient ideal, algebraic geometry, SOSTOOLS.

\section{Introduction}

We consider the global optimization problem

$$
f^{*}=\min _{x \in \mathbb{R}^{n}} f(x)
$$

where $x=\left(x_{1}, \ldots, x_{n}\right) \in \mathbb{R}^{n}$ is a real vector, and $f(x)$ is a multivariate polynomial of degree d. As is well-known, the optimization problem (1.1) is NP-hard even when $d$ is fixed to be four 15]. A lower bound can be computed efficiently using the Sum Of Squares (SOS) relaxation

$$
f_{\text {sos }}^{*}=\operatorname{maximize} \gamma \text { subject to } f(x)-\gamma \succeq_{\text {sos }} 0,
$$

where the inequality $g \succeq_{\text {sos }} 0$ means that the polynomial $g$ is SOS, i.e. a sum of squares of other polynomials. We refer to [13, 16, 17, 18, 19, for introductions to SOS techniques and their applications. SOS methods solve 1.2 in polynomial time, provided either $n$ or $d$ is fixed, by reducing the problem to solving a Semidefinite Program (SDP, see 24 for an introduction). The relationship between (1.1) and (1.2) is as follows: $f_{\text {sos }}^{*} \leq f^{*}$ and the equality holds if and only if $f(x)-f^{*}$ is SOS.

\footnotetext{
*demmel@cs. berkeley.edu, Department of Mathematics and EECS, University of California, Berkeley, CA 94720.

$\dagger$ Corresponding author, njw@math. berkeley.edu, Dept. of Mathematics, Univ. of California, Berkeley, CA 94720.

${ }^{\ddagger}$ bernd@math. berkeley.edu, Department of Mathematics, University of California, Berkeley, CA 94720.
} 
Blekherman 3 recently showed that, for fixed even degree $d \geq 4$, the ratio between the volume of all nonnegative polynomials and the volume of all SOS polynomials tends to infinity when $n$ goes to infinity. In other words, for large $n$, there are many more nonnegative polynomials than SOS polynomials. This contrasts with the experimental results of Parrilo and Sturmfels [18 who used (1.2) to exactly solve optimization problems (1.1) which were drawn at random from a natural distribution. Their test family will be revisited in Section 6.1.

For dealing with the challenging case when $f_{\text {sos }}^{*}$ is strictly less than $f^{*}$, Lasserre [13] proposed finding a sequence of lower bounds for $f(x)$ in some large ball $\left\{x \in \mathbb{R}^{n}:\|x\|_{2} \leq R\right\}$. His approach is based on the result [1] that SOS polynomials are dense among polynomials which are nonnegative on some compact set. This sequence converges to $f^{*}$ when the degrees of the polynomials introduced in the algorithm go to infinity. But it may not converge in finitely many steps, and the degrees of the required auxiliary polynomials can be very large.

In this paper, we introduce a method which can find the global minimum and terminate in finitely many steps, under some mild assumptions. Our point of departure is the observation that all local minima and global minima of 1.1. occur at points in the real gradient variety

$$
V_{\text {grad }}^{\mathbb{R}}(f)=\left\{u \in \mathbb{R}^{n}:(\nabla f)(u)=0\right\} .
$$

The gradient ideal of $f$ is the ideal in $\mathbb{R}\left[x_{1}, \ldots, x_{n}\right]$ generated by all partial derivatives of $f$ :

$$
\mathcal{I}_{\text {grad }}(f)=\langle\nabla f(x)\rangle=\left\langle\frac{\partial f}{\partial x_{1}}, \frac{\partial f}{\partial x_{2}}, \cdots, \frac{\partial f}{\partial x_{n}}\right\rangle .
$$

There are several recent references on minimizing polynomials by way of the gradients. Hanzon and Jibetean [10] suggest applying perturbations to $f$ to produce a sequence of polynomials $f_{\lambda}$ (for small $\lambda$ ) with the property that the gradient variety of $f_{\lambda}$ is finite and the minima $f_{\lambda}^{*}$ converge to $f^{*}$ as $\lambda$ goes to 0. Laurent 14] and Parrilo [21] discuss the more general problem of minimizing a polynomial subject to polynomial equality constraints (not necessarily partial derivatives). Under the assumption that the variety defined by the equations is finite, the matrix method proposed in [14 has finite convergence even if the ideal generated by the constraints is not radical. Building on 10, 14, Jibetean and Laurent [1] propose to compute $f^{*}$ by solving a single SDP, provided the gradient variety is finite (radicalness is not necessary).

The approach of this paper is to find a lower bound $f_{\text {grad }}^{*}$ for (1.1) by requiring $f-f_{\text {grad }}^{*}$ to be SOS on the real gradient variety $V_{\text {grad }}^{\mathbb{R}}(f)$ instead of $\mathbb{R}^{n}$. Under the assumption that the infimum $f^{*}$ is attained, we can find a monotonically increasing sequence $\left\{f_{N, \text { grad }}^{*}\right\}$ such that $\lim _{N \rightarrow \infty} f_{N, \text { grad }}^{*}=f^{*}$, and the equality $f_{N, \text { grad }}^{*}=f^{*}$ holds (i.e., finite convergence) for some large integer $N$ when the ideal $\mathcal{I}_{\text {grad }}(f)$ is radical which is usual in practice.

This paper is organized as follows. Section 2 offers a review of fundamental results from (real) algebraic geometry. In Section 3 we prove that a positive polynomial is SOS modulo its gradient ideal. The same holds for non-negative polynomials if the gradient ideal is radical. The resulting algorithms for unconstrained polynomial minimization will be presented in Section 4. Section 5 generalizes our methods to constrained optimization. In Section 6 we discuss numerical experiments using the software SOSTOOLS. Section 7 draws some conclusions.

\section{Tools from Algebraic Geometry}

This section will introduce some basic notions from algebraic geometry needed for our discussion. Readers may consult [6, 7] 8] for more details. We write $\mathbb{R}[x]=\mathbb{R}\left[x_{1}, \ldots, x_{n}\right]$ for the ring of all polynomials in $n$ variables $x=\left(x_{1}, \ldots, x_{n}\right)$ with real coefficients. A subset $I$ of $\mathbb{R}[x]$ is an $i d e a l$ if $p \cdot h \in I$ for any $p \in I$ and $h \in \mathbb{R}[x]$. If $g_{1}, \ldots, g_{r} \in \mathbb{R}[x]$ then $\left\langle g_{1}, \cdots, g_{m}\right\rangle$ denotes 
the smallest ideal containing the $g_{i}$. Equivalently, $\left\langle g_{1}, \cdots, g_{m}\right\rangle$ is the set of all polynomials that are polynomial linear combinations of the $g_{i}$. Every ideal arises in this way:

Theorem 2.1 (Hilbert Basis Theorem, Section 5, Ch. 2, 6])

Every ideal $I \subset \mathbb{R}[x]$ has a finite generating set, i.e., $I=\left\langle g_{1}, \cdots, g_{m}\right\rangle$ for some $g_{1}, \cdots, g_{m} \in I$.

The variety of an ideal $I$ is the set of all common complex zeros of the polynomials in $I$ :

$$
V(I)=\left\{x \in \mathbb{C}^{n}: p(x)=0 \text { for all } p \in I\right\} .
$$

The subset of all real points in $V(I)$ is the real variety of $I$. It is denoted

$$
V^{\mathbb{R}}(I)=\left\{x \in \mathbb{R}^{n}: p(x)=0 \text { for all } p \in I\right\} .
$$

If $I=\left\langle g_{1}, \ldots, g_{m}\right\rangle$ then $V(I)=V\left(g_{1}, \ldots, g_{m}\right)=\left\{x \in \mathbb{C}^{n}: g_{1}(x)=\cdots=g_{m}(x)=0\right\}$. An ideal $I \subseteq \mathbb{R}[x]$ is zero-dimensional if its variety $V(I)$ is a finite set. This condition is much stronger than requiring that the real variety $V^{\mathbb{R}}(I)$ be a finite set. For instance, $I=\left\langle x_{1}^{2}+x_{2}^{2}\right\rangle$ is not zero-dimensional: the real variety $V^{\mathbb{R}}(I)=\{(0,0)\}$ is only one point of the curve $V(I)$.

Theorem 2.2 (Chapter 5, [6]) The following conditions are equivalent for an ideal $I \subset \mathbb{R}[x]$ :

(i) I is zero-dimensional (the variety $V(I)$ is a finite set);

(ii) the quotient ring $\mathbb{R}[x] / I$ is a finite-dimensional $\mathbb{R}$-vector space;

(iii) if $G$ is a Gröbner basis of $I$, then for each $1 \leq i \leq n$, there exists an integer $m_{i} \geq 0$ such that $x_{i}^{m_{i}}$ is the leading term of some $g \in G$.

A variety $V \subseteq \mathbb{C}^{n}$ is irreducible if there do not exist two proper subvarieties $V_{1}, V_{2} \varsubsetneqq V$ such that $V=V_{1} \cup V_{2}$. Given a variety $V \subseteq \mathbb{C}^{n}$, the set of all polynomials that vanish on $V$ is an ideal

$$
I(V)=\{p \in \mathbb{R}[x]: p(u)=0 \text { for all } u \in V\} .
$$

Given any ideal $I$ of $\mathbb{R}[x]$, its radical is the ideal

$$
\sqrt{I}=\left\{q \in \mathbb{R}[x]: q^{m} \in I \text { for some } m \in \mathbb{N}\right\} .
$$

Note that $I \subseteq \sqrt{I}$. We say that $I$ is a radical ideal if $\sqrt{I}=I$. Clearly, the ideal $I(V)$ defined by a variety $V$ is a radical ideal. The following theorems offer a converse to this observation:

Theorem 2.3 (Hilbert's Weak Nullstellensatz)

If $I$ is an ideal in $\mathbb{R}[x]$ such that $V(I)=\emptyset$ then $1 \in I$.

Theorem 2.4 (Hilbert's Strong Nullstellensatz)

If $I$ is an ideal in $\mathbb{R}[x]$ then $I(V(I))=\sqrt{I}$.

In real algebraic geometry, we are also interested in subsets of $\mathbb{R}^{n}$ of the form

$$
S=\left\{x \in \mathbb{R}^{n}: g_{1}(x)=\cdots=g_{m}(x)=0, h_{1}(x) \geq 0, \cdots, h_{\ell}(x) \geq 0\right\},
$$

where $g_{i}, h_{j} \in \mathbb{R}[x]$. We call $S$ a basic semi-algebraic set. With the given description of $S$, we associate the following set of polynomials:

$$
M(S)=\left\{\sigma_{0}(x)+\sum_{i=1}^{m} \lambda_{i}(x) g_{i}(x)+\sum_{j=1}^{\ell} h_{j}(x) \sigma_{j}(x): \sigma_{0}, \cdots, \sigma_{\ell} \text { are SOS, } \lambda_{i}(x) \in \mathbb{R}[x]\right\} .
$$

Theorem 2.5 (Putinar, 22]) Assume that the basic semi-algebraic set $S$ is compact and there exists one polynomial $\rho(x) \in M(S)$ such that the set $\left\{x \in \mathbb{R}^{n}: \rho(x) \geq 0\right\}$ is compact. Then every polynomial $p(x)$, which is positive on $S$, belongs to $M(S)$. 
Suppose we are given an ideal $I=\left\langle h_{1}, \ldots, h_{r}\right\rangle$ in $\mathbb{R}[x]$ and a polynomial $f \in \mathbb{R}[x]$. Then we can regard $f$ as an element in the quotient $\mathbb{R}[x] / I$. Even if $f$ is not SOS in $\mathbb{R}[x]$, it is possible for $f$ to be SOS in the quotient ring $\mathbb{R}[x] / I$. For $f$ to be SOS in $\mathbb{R}[x] / I$ means that there exists a $q \in I$ such that $f-q$ is $\operatorname{SOS}$ in $\mathbb{R}[x]$, or, more explicitly, that $f$ has a representation

$$
f(x)=\sum_{j} q_{j}^{2}(x)+\sum_{i} \phi_{i}(x) h_{i}(x)
$$

for some polynomials $q_{j}(x)$ and $\phi_{i}(x)$, Clearly, if $f$ is SOS in $\mathbb{R}[x] / I$ then the function $f(x)$ is non-negative on the real variety $V^{\mathbb{R}}(I)$. The following partial converse holds if $V^{\mathbb{C}}(I)$ is finite.

Theorem 2.6 (Parrilo, [21]) Let I be a zero-dimensional radical ideal in $\mathbb{R}[x]$. Then a polynomial $f \in \mathbb{R}[x]$ is nonnegative on the real variety $V^{\mathbb{R}}(I)$ if and only if $f(x)$ is $S O S$ in $\mathbb{R}[x] / I$.

We close with the following theorem, which is a special case of the real Nullstellensatz.

Theorem 2.7 (Real Nullstellensatz, [2, 4, 5.) Let I be an ideal in $\mathbb{R}[x]$ whose real variety $V^{\mathbb{R}}(I)$ is empty. Every polynomial $f(x)$ is $S O S$ in $\mathbb{R}[x] / I$. In particular, -1 is $S O S$ in $\mathbb{R}[x] / I$.

\section{Polynomials Over Their Gradient Varieties}

Consider a polynomial $f \in \mathbb{R}[x]$ and its gradient ideal $\mathcal{I}_{\text {grad }}(f)$ as in (1.4). A natural idea in solving (1.1) is to apply Theorem 2.6 to the ideal $I=\mathcal{I}_{\text {grad }}(f)$, since the minimum of $f$ over $\mathbb{R}^{n}$ will be attained at a subset of $V^{\mathbb{R}}(I)$ if it is attained at all. However, the hypothesis of Theorem 2.6] requires that $I$ be zero-dimensional, which means that the complex variety $V_{\text {grad }}(f)=V(I)$ of all critical points must be finite. Our results in this section remove this restrictive hypothesis. We shall prove that every nonnegative $f$ is SOS in $\mathbb{R}[x] / I$ as long as the gradient ideal $I=\mathcal{I}_{\text {grad }}(f)$ is radical.

Theorem 3.1 Assume that the gradient ideal $\mathcal{I}_{\text {grad }}(f)$ is radical. If the real polynomial $f(x)$ is nonnegative over $V_{g r a d}^{\mathbb{R}}(f)$, then there exist real polynomials $q_{i}(x)$ and $\phi_{j}(x)$ so that

$$
f(x)=\sum_{i=1}^{s} q_{i}(x)^{2}+\sum_{j=1}^{n} \phi_{j}(x) \frac{\partial f}{\partial x_{j}} .
$$

The proof of this theorem will be based on the following two lemmas. The first is a generalization of the Lagrange Interpolation Theorem from sets of points to disjoint varieties.

Lemma 3.2 Let $V_{1}, \ldots, V_{r}$ be pairwise disjoint varieties in $\mathbb{C}^{n}$. Then there exist polynomials $p_{1}, \ldots, p_{r} \in \mathbb{R}[x]$ such that $p_{i}\left(V_{j}\right)=\delta_{i j}$, where $\delta_{i j}$ is the Kronecker delta function.

Proof Our definition of variety requires that each $V_{j}$ is actually defined by polynomials with real coefficients. If $I_{j}=I\left(V_{j}\right)$ is the radical ideal of $V_{j}$ then we have $V_{j}=V\left(I_{j}\right)$. Fix an index $j$ and let $W_{j}$ denote the union of the varieties $V_{1}, \ldots, V_{i-1}, V_{i+1}, \ldots, V_{r}$. Then

$$
I\left(W_{j}\right) \quad=\quad I_{1} \cap \cdots \cap I_{j-1} \cap I_{j+1} \cap \cdots \cap I_{r} .
$$

Our hypothesis implies that $V_{j} \cap W_{j}=\emptyset$. By Hilbert's Weak Nullstellensatz (Theorem [2.3], there exist polynomials $p_{j} \in I\left(W_{j}\right)$ and $q_{j} \in I_{j}$ such that $p_{j}+q_{j}=1$. This identity shows that $p_{j}\left(V_{j}\right)=1$ and $p_{j}\left(V_{k}\right)=0$ for $k \neq j$. Hence the $r$ polynomials $p_{1}, \ldots, p_{r}$ have the desired properties.

Now consider the behavior of the polynomial $f(x)$ over its gradient variety $V_{\text {grad }}(f)$. We make use of the fact that $V_{\text {grad }}(f)$ is a finite union of irreducible subvarieties $\left.([2] \S 2]\right)$. 
Lemma 3.3 Let $W$ be an irreducible subvariety of $V_{\text {grad }}(f)$ and suppose that $W$ contains at least one real point. Then $f(x)$ is constant on $W$.

Proof If we replace our polynomial ring $\mathbb{R}[x]$ by $\mathbb{C}[x]$ then $W$ either remains irreducible or it becomes a union of two irreducible components $W=W_{1} \cup W_{2}$ which are exchanged under complex conjugation. Let us first consider the case when $W$ is irreducible in the Zariski topology induced from $\mathbb{C}[x]$. Then $W$ is connected in the strong topology on $\mathbb{C}^{n}$. In fact, $W$ is path-connected. Let $x, y$ be two arbitrary points in $W$. There exists a smooth path $\varphi(t)(0 \leq t \leq 1)$ (or piecewise smooth path) lying inside $W$ such that $x=\varphi(0)$ and $y=\varphi(1)$. By the Mean Value Theorem of Calculus, it holds that for some $t^{*} \in(0,1)$

$$
f(y)-f(x)=\nabla f\left(\varphi\left(t^{*}\right)\right)^{T} \varphi^{\prime}\left(t^{*}\right)=0
$$

since $\nabla f$ vanishes on $W$. We conclude that $f(x)=f(y)$, and hence $f$ is constant on $W$.

Now consider the case when $W=W_{1} \cup W_{2}$ where $W_{1}$ and $W_{2}$ are exchanged by complex conjugation. We had assumed that $W$ contains a real point $p$. Since $p$ is fixed under complex conjugation, $p \in W_{1} \cap W_{2}$. By the same argument as above, $f(x)=f(p)$ for all $x \in W$.

Proof of Theorem 3.1] Consider the irreducible decomposition of $V_{\text {grad }}(f)$. We group together all components which have no real point and all components on which $f$ takes the same real value. Hence the gradient variety has a decomposition

$$
V_{\text {grad }}(f)=W_{0} \cup W_{1} \cup W_{2} \cup \cdots \cup W_{r},
$$

such that $W_{0}$ has no real point and $f$ is a real constant on each other variety $W_{i}$, say,

$$
f\left(W_{1}\right)>f\left(W_{2}\right)>\cdots>f\left(W_{r}\right) \geq 0 .
$$

The varieties $W_{i}$ are pairwise disjoint, so by Lemma 3.2 there exist polynomials $p_{i} \in \mathbb{R}[x]$ such that $p_{i}\left(W_{j}\right)=\delta_{i j}$. By Theorem $[2.7$ there exists a sum of squares $\operatorname{sos}(x) \in \mathbb{R}[x]$ such that $f(x)=\operatorname{sos}(x)$ for all $x \in W_{0}$. Using the non-negative real numbers $\alpha_{j}:=\sqrt{f\left(W_{j}\right)}$, we define

$$
q(x)=\operatorname{sos}(x) \cdot p_{0}^{2}(x)+\sum_{i=1}^{r}\left(\alpha_{i} \cdot p_{i}(x)\right)^{2} .
$$

By construction, $f(x)-q(x)$ vanishes on the gradient variety $V_{\text {grad }}(f)$. The gradient ideal $\mathcal{I}_{\text {grad }}(f)$ was assumed to be radical. Using Hilbert's Strong Nullstellensatz (Theorem 2.4), we conclude that $f(x)-q(x)$ lies in $\mathcal{I}_{\text {grad }}(f)$. Hence the desired representation (3.1) exists.

In Theorem 3.1 the assumption that $\mathcal{I}_{\text {grad }}(f)$ is radical cannot be removed. This is shown by the following counterexample which was suggested to us by Claus Scheiderer.

Example 3.4 Let $n=3$ and consider the polynomial

$$
f(x, y, z)=x^{8}+y^{8}+z^{8}+M(x, y, z)
$$

where $M(x, y, z)=x^{4} y^{2}+x^{2} y^{4}+z^{6}-3 x^{2} y^{2} z^{2}$ is the Motzkin polynomial, which is is nonnegative but not a sum of squares. The residue $\operatorname{ring} A=\mathbb{R}[x, y, z] / \mathcal{I}_{\text {grad }}(f)$ is a real vector space of dimension $7^{3}=243$ because the three partial derivatives form a Gröbner basis:

$$
\begin{aligned}
& \frac{\partial f}{\partial x}=\underline{8 x^{7}}+4 x^{3} y^{2}+2 x y^{4}-6 x y^{2} z^{2} \\
& \frac{\partial f}{\partial y}=\underline{8 y^{7}}+2 x^{4} y+4 x^{2} y^{3}-6 x^{2} y z^{2} \\
& \frac{\partial f}{\partial z}=\underline{8 z^{7}}+6 z^{5}-6 x^{2} y^{2} z .
\end{aligned}
$$


Reduction modulo this Gröbner basis shows that $f(x, y, z)$ is congruent to $\frac{1}{4} M(x, y, z) \bmod -$ ulo $\mathcal{I}_{\text {grad }}(f)$. Hence it suffices to show that $M(x, y, z)$ is not a sum of squares in $A$. Suppose otherwise. Then there exist polynomials $s_{i}, \phi_{1}, \phi_{1}, \phi_{3} \in \mathbb{R}[x, y, z]$ such that

$$
M(x, y, z)=\sum_{i} s_{i}^{2}+\frac{\partial f}{\partial x} \phi_{1}(x, y, z)+\frac{\partial f}{\partial y} \phi_{2}(x, y, z)+\frac{\partial f}{\partial z} \phi_{3}(x, y, z) .
$$

By inspecting $\frac{\partial f}{\partial x}, \frac{\partial f}{\partial y}, \frac{\partial f}{\partial z}$ and $M$, we see that every monomial in the expansion of $\sum_{i} s_{i}^{2}$ has degree at least six, and the monomials $x^{6}, y^{6}, x^{4} z^{2}, y^{4} z^{2}, x^{2} z^{4}, y^{2} z^{4}$ cannot occur. This implies

$$
\begin{aligned}
s_{i}(x, y, z) & =A_{1}^{(i)} x y^{2}+A_{2}^{(i)} x^{2} y+A_{3}^{(i)} z^{3}+A_{4}^{(i)} x y z+\text { higher order terms } \\
\phi_{1}(x, y, z) & =B x+\text { other linear and high order terms } \\
\phi_{2}(x, y, z) & =C y+\text { other linear and high order terms } \\
\phi_{3}(x, y, z) & =D z+\text { other linear and high order terms. }
\end{aligned}
$$

Comparing the terms in $M(x, y, z)$ with the expansion of the right hand side in (3.4), we get

$$
\begin{aligned}
x^{4} y^{2}: & 1 & =\sum_{i} A_{1}^{(i)^{2}}+4 B+2 C \\
x^{2} y^{4}: & 1 & =\sum_{i} A_{2}^{(i)^{2}}+2 B+4 C \\
z^{6}: & 1 & =\sum_{i} A_{3}^{(i)^{2}}+6 D \\
x^{2} y^{2} z^{2}: & -3 & =\sum_{i} A_{4}^{(i)^{2}}-6 B-6 C-6 D .
\end{aligned}
$$

Summing the above equations together results in

$$
0=\sum_{i} A_{1}^{(i)^{2}}+\sum_{i} A_{2}^{(i)^{2}}+\sum_{i} A_{3}^{(i)^{2}}+\sum_{i} A_{4}^{(i)^{2}} .
$$

Thus $A_{1}^{(i)}=A_{2}^{(i)^{2}}=A_{3}^{(i)^{2}}=A_{4}^{(i)}=0$ and $B=C=D=\frac{1}{6}$. Hence $s_{i}$ only contains terms of degree $\geq 4$. Let $E^{(i)}$ be the coefficient of $z^{4}$ in $s_{i}(x, y, z)$. Comparing the coefficient of $z^{8}$ in (3.4), we get $0=\sum_{i} E^{(i)^{2}}+\frac{8}{6}$, which is a contradiction. We conclude that the nonnegative polynomial $f(x, y, z)=M(x, y, z)+x^{8}+y^{8}+z^{8}$ is not SOS modulo its gradient ideal.

In cases (like Example 3.4 when the gradient ideal is not radical, the following still holds.

Theorem 3.5 Let $f(x) \in \mathbb{R}[x]$ be a polynomial which is strictly positive on its real gradient variety $V_{\text {grad }}^{\mathbb{R}}(f)$, Then $f(x)$ is SOS modulo its gradient ideal $\mathcal{I}_{\text {grad }}(f)$.

Proof We retain the notation from the proof of Theorem 3.1 The decomposition (3.2) gives rise to a decomposition of $\mathcal{I}_{\text {grad }}(f)=J_{0} \cap J_{1} \cap \cdots \cap J_{r}$, where $W_{i}=V\left(J_{i}\right)$ and $J_{i}+J_{k}=\mathbb{R}[x]$ for $i \neq k$. By the Chinese Remainder Theorem [8], we have

$$
\mathbb{R}[x] / \mathcal{I}_{\text {grad }}(f) \simeq \mathbb{R}[x] / J_{0} \times \mathbb{R}[x] / J_{1} \times \cdots \times \mathbb{R}[x] / J_{r} .
$$

Here $V^{\mathbb{R}}\left(J_{0}\right)=\emptyset$. Hence, by Theorem [2.7 there exists a sum of squares $\operatorname{sos}(x) \in \mathbb{R}[x]$ such that $f(x)-\operatorname{sos}(x) \in J_{0}$. By assumption, $\alpha_{i}^{2}=f\left(W_{i}\right)$ is strictly positive for all $i \geq 1$. The polynomial $f(x) / \alpha_{i}^{2}-1$ vanishes on $W_{i}$. By Hilbert's Strong Nullstellensatz, there exists an integer $m>0$ such that $\left(f(x) / \alpha_{i}^{2}-1\right)^{m}$ is in the ideal $J_{i}$. We construct a square root of 
$f(x) / \alpha_{i}^{2}$ in the residue ring $\mathbb{R}[x] / J_{i}$ using the familiar Taylor series expansion for the square root function:

$$
\left(1+\left(f(x) / \alpha_{i}^{2}-1\right)\right)^{1 / 2}=\sum_{k=0}^{m-1}\left(\begin{array}{c}
1 / 2 \\
k
\end{array}\right)\left(f(x) / \alpha_{i}^{2}-1\right)^{k} \bmod J_{i} .
$$

Multiplying this polynomial by $\alpha_{i}$, we get a polynomial $q_{i}(x)$ such that $f(x)-q_{i}^{2}(x)$ is in the ideal $J_{i}$. We have shown that $f(x)$ maps to the vector $\left(\operatorname{sos}(x), q_{1}(x)^{2}, q_{2}(x)^{2}, \ldots, q_{r}(x)^{2}\right)$ under the isomorphism (3.5). That vector is clearly a sum of squares in the ring on the right hand side of 3.5 . We conclude that $f(x)$ is a sum of squares in $\mathbb{R}[x] / \mathcal{I}_{\text {grad }}(f)$.

Example 3.6 Let $f$ be the polynomial in Example 3.4 and let $\epsilon$ be any positive constant. Theorem 3.5 says that $f+\epsilon$ is SOS modulo $\mathcal{I}_{\text {grad }}(f)$. Such a representation can be found by symbolic computation as follows. Primary decomposition over $\mathbb{Q}[x, y, z]$ yields

$$
\mathcal{I}_{\text {grad }}(f)=J_{0} \cap J_{1},
$$

where $V^{\mathbb{R}}\left(J_{0}\right)=\emptyset$ and and $\sqrt{J_{1}}=\langle x, y, z\rangle$. The ideal $J_{1}$ has multiplicity 153 , and it contains the square $f^{2}$ of our given polynomial. The ideal $J_{0}$ has multiplicity 190 . Its variety $V\left(J_{0}\right)$ consists of 158 distinct points in $\mathbb{C}^{3}$. By elimination, we can reduce to the univariate case. Using the algorithm of 4$] 5$ for real radicals in $\mathbb{Q}[z]$, we find a sum of squares $\operatorname{sos}(z) \in \mathbb{Q}[z]$ such that $f-\operatorname{sos}(z) \in J_{0}$. Running Buchberger's algorithm for $J_{0}+J_{1}=\langle 1\rangle$, we get polynomials $p_{0} \in J_{0}$ and $p_{1} \in J_{1}$ such that $p_{0}+p_{1}=1$. The following polynomial is a sum of squares,

$$
p_{1}^{2} \cdot(\operatorname{sos}(z)+\epsilon)+p_{0}^{2} \cdot \epsilon \cdot\left(1+\frac{1}{2 \epsilon} f\right)^{2},
$$

and it is congruent to $f(x, y, z)+\epsilon$ modulo $\mathcal{I}_{\text {grad }}(f)=J_{0} \cap J_{1}=J_{0} \cdot J_{1}$. Note that the coefficients of the right hand polynomial in the SOS representation (3.6) tend to infinity as $\epsilon$ approaches zero. This is consistent with the conclusion of Example 3.4

\section{Unconstrained Optimization}

This section concerns finding the global minimum of a polynomial function $f(x)$ on $\mathbb{R}^{n}$. Let $\mathbb{R}[x]_{m}$ denote the $\left(\begin{array}{c}n+m \\ m\end{array}\right)$-dimensional vector space of polynomials of degree at most $m$. Since local or global minima occur only when the gradient is zero, we consider the SOS problem

$$
\text { Maximize } \gamma \text { subject to } f(x)-\gamma-\sum_{j=1}^{n} \phi_{j}(x) \frac{\partial f}{\partial x_{j}} \succeq_{\text {sos }} 0 \text { and } \phi_{j}(x) \in \mathbb{R}[x]_{2 N-d+1} \text {. }
$$

Here $d$ is the degree of polynomial $f(x)$, and $N$ is an integer to be chosen by the user. Let $f_{N, \text { grad }}^{*}$ denote the optimal value $\gamma$ of the optimization problem 4.1. This is a lower bound for the global minimum $f^{*}$ of the polynomial $f(x)$. The lower bound gets better as $N$ increases:

$$
\cdots \leq f_{N-1, \text { grad }}^{*} \leq f_{N, \text { grad }}^{*} \leq f_{N+1, \text { grad }}^{*} \leq \cdots \leq f^{*} .
$$

\subsection{SOS Optimization using the Software SOSTOOLS}

The problem 4.1 is a standard SOS program. It can be translated into an SDP as described in 17 18, 19. The decision variables in (4.1) are the real number $\gamma$ and the coefficients the multiplier polynomials $\phi_{j}(x)$. The resulting SDP is dual to the formulation of Lasserre 13. The SOS program (4.1) can be solved using the software package SOSTOOLS. We refer to [20] for the documentation. For instance, if we take $N=4$ and $f(x, y, z)$ the trivariate polynomial in Example 3.4 then 4.1] translates into an SOSTOOLS program as follows: 


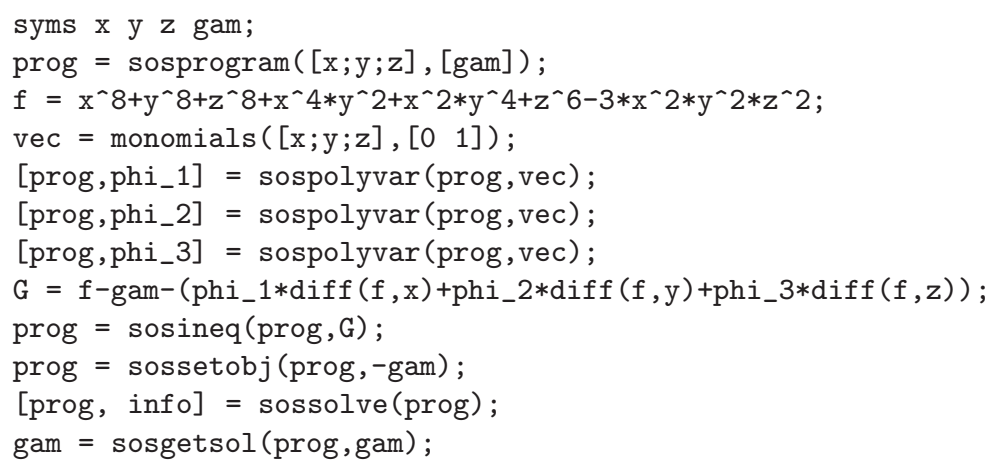

The system returns the following lower bound $\gamma=f_{4, \text { grad }}^{*}$ for the global minimum $f^{*}=0$ : gam $=-.12077 \mathrm{e}-8$

Even if we increase the value of $N$, the lower bound $f_{N, \text { grad }}^{*}$ always remains negative, since $f$ is not SOS modulo its gradient ideal. However, the sequence $\left\{f_{N, \text { grad }}^{*}\right\}_{N \geq 4}$ converges to zero.

\subsection{Convergence of the Lower Bounds}

We have the following general result concerning the convergence of the lower bounds.

Theorem 4.1 Let $f(x)$ be a polynomial in $n$ real variables which attains its infimum $f^{*}$ over $\mathbb{R}^{n}$. Then $\lim _{N \rightarrow \infty} f_{N, \text { grad }}^{*}=f^{*}$. Furthermore, if the gradient ideal $\mathcal{I}_{\text {grad }}(f)$ is radical, then $f^{*}$ is attainable, i.e., there exist an integer $N$ such that $f_{N, \text { grad }}^{*}=f^{*}$.

Proof Since $f(x)$ attains its infimum, the global minima of $f(x)$ must occur on the real gradient variety $V_{\text {grad }}^{\mathbb{R}}(f)$. It is obvious that any real number $\gamma$ which satisfies the SOS constraint in (4.1) is a lower bound of $f(x)$, and we have the sequence of inequalities in (4.2). Consider an arbitrary small real number $\varepsilon>0$. The polynomial $f(x)-f^{*}+\varepsilon$ is strictly positive on its real gradient variety $V_{\text {grad }}^{\mathbb{R}}(f)$. By Theorem[3.5 $f(x)-f^{*}+\varepsilon$ is SOS modulo $\mathcal{I}_{\text {grad }}(f)$. Hence there exists an integer $N(\epsilon)$ such that

$$
f_{N, \text { grad }}^{*} \geq f^{*}-\varepsilon \quad \text { for all } \quad N \geq N(\epsilon) .
$$

Since the sequence $\left\{f_{N, \text { grad }}^{*}\right\}$ is monotonically increasing, it follows that $\lim _{N \rightarrow \infty} f_{N, \text { grad }}^{*}=f^{*}$.

Now suppose $\mathcal{I}_{\text {grad }}(f)=\mathcal{I}_{\text {grad }}\left(f-f^{*}\right)$ is a radical ideal. The nonnegative polynomial $f(x)-f^{*}$ is SOS modulo $\mathcal{I}_{\text {grad }}(f)$ by Theorem 3.1 Hence $f_{N, \text { grad }}^{*}=f^{*}$ for some $N \in \mathbb{Z}_{>0}$.

Remarks: (i) The condition that $f(x)$ attains its infimum cannot be removed. Otherwise the infimum $f_{\text {grad }}^{*}$ of $f(x)$ on $V_{\text {grad }}^{\mathbb{R}}(f)$ need not be a lower bound for $f(x)$ on $\mathbb{R}^{n}$. A counterexample is $f(x)=x^{3}$. Obviously $f(x)$ has infimum $f^{*}=-\infty$ on $\mathbb{R}^{1}$. However, $f_{\text {grad }}^{*}=f_{\text {grad }, N}^{*}=0$ for all $N \geq 1$ because $f(x)=\left(\frac{x}{3}\right) f^{\prime}(x)$ is in the gradient ideal $\mathcal{I}_{\text {grad }}(f)=\left\langle f^{\prime}(x)\right\rangle$.

(ii) It is also not always the case that $f_{\text {grad }}^{*}=f^{*}$ when $f^{*}$ is finite. Consider the bivariate polynomial $f(x, y)=x^{2}+(1-x y)^{2}$. We can see that $f^{*}=0$ is not attained, but $f_{\text {grad }}^{*}=1>f^{*}$.

(iii) If $f(x)$ attains its infimum but $\mathcal{I}_{\text {grad }}(f)$ is not radical, we have only that $\lim _{N \rightarrow \infty} f_{N, \text { grad }}^{*}=$ $f^{*}$. But there is typically no integer $N$ with $f_{N, \text { grad }}^{*}=f^{*}$, as shown in Example 3.4 .

\subsection{Duality and an Algorithm}

In this subsection, we describe a dual formulation of the SOS problem 4.1], and we present an explicit algorithm for finding the global minimizer of a polynomial $f(x)$. At first, we 
introduce some notation. Given any polynomial $p(x)$ in $\mathbb{R}[x]_{m}$, we write $p(x)=\sum_{|\alpha| \leq m} p_{\alpha} x^{\alpha}$ where $\alpha \in \mathbb{Z}_{\geq 0}^{n}$ and $|\alpha|=\sum_{j=1}^{n} \alpha_{j}$. In what follows, we denote by $p \in \mathbb{R}^{\left(\begin{array}{c}n+m \\ m\end{array}\right)}$ the vector of coefficients $p_{\alpha}$ of $p(x)$. For any integer $N$, we write $\mathbb{Z}_{N}^{n}=\left\{\tau \in \mathbb{Z}_{>0}^{n}:|\tau| \leq N\right\}$ and we denote by $\operatorname{mon}_{N}(x)$ the column vector of monomials of degree up to $N$, i.e.,

$$
\operatorname{mon}_{N}(x)=\left(1, x_{1}, \cdots, x_{n}, x_{1}^{2}, x_{1} x_{2}, \cdots, x_{1}^{N}, \cdots, x_{n}^{N}\right)^{T} .
$$

The dimension of $\operatorname{mon}_{N}(x)$ is the binomial coefficient $\left(\begin{array}{c}n+N \\ N\end{array}\right)$. Given any finite or infinite vector $y=\left(y_{\alpha}\right)$, indexed by integer vectors $\alpha \in \mathbb{Z}_{\geq 0}^{n}$, define $M_{N}(y)$ to be its moment matrix

$$
M_{N}(y)=\left(y_{\alpha+\beta}\right)_{\alpha, \beta \in \mathbb{Z}_{N}^{n}} .
$$

The moment matrix represents the linear map $p \mapsto p * y$, where, for any polynomial $p(x)=$ $\sum_{\beta} p_{\beta} x^{\beta}$, the vector $p * y$ has coordinates $(p * y)_{\alpha}=\sum_{\beta} p_{\beta} y_{\alpha+\beta}$.

Let $f(x)$ be the polynomial we wish to minimize. Its vector of coefficients is $f$. Let $f_{i}$ denote the vector of coefficients of the $i$-th partial derivative $\frac{\partial f}{\partial x_{i}}$. We rewrite 4.1] as follows:

$$
\begin{aligned}
& f_{N, \text { grad }}^{*}=\max _{\substack{\gamma \in \mathbb{R}, \sigma \in \mathbb{R}[x]_{2 N} \\
\phi_{j}(x) \in \mathbb{R}[x]_{2 N-d+1}}} \gamma \quad \text { subject to } \sigma(x) \succeq_{\text {sos }} 0 \\
& \text { and } f(x)-\gamma=\sigma(x)+\sum_{j=1}^{n} \phi_{j}(x) \frac{\partial f}{\partial x_{j}} .
\end{aligned}
$$

We call this formulation of our problem the dual $S D P$, because it is dual to the formulation proposed in 13 14. The corresponding primal SDP supposes that $d$ is even and it is given by

$$
\begin{aligned}
f_{N, \text { mom }}^{*}=\min _{y} & f^{T} y \\
\text { s.t. } & M_{N-d / 2}\left(f_{i} * y\right)=0, i=1, \cdots, n \\
& M_{N}(y) \succeq 0, y_{0}=1 .
\end{aligned}
$$

The following theorem relates the primal and dual objective function values $f_{N, m o m}^{*}$ and $f_{N, \text { grad }}^{*}$, and it shows how to extract a point in $\mathbb{R}^{n}$ at which the minimum of $f(x)$ is attained.

Theorem 4.2 Assume $f(x)$ attains its infimum $f^{*}$ over $\mathbb{R}^{n}$ (hence $d$ is even). Then we have:

(i) $f_{N, m o m}^{*}=f_{N, \text { grad }}^{*}$ and hence $\lim _{N \rightarrow \infty} f_{N, m o m}^{*}=f^{*}$. This means that the strong duality holds.

(ii) Suppose $f_{N, \text { grad }}^{*}=f^{*}$ for some $N$. If $x^{*} \in \mathbb{R}^{n}$ minimizes $f(x)$, then $y^{*}=$ mon $_{2 N}\left(x^{*}\right) \in$ $\mathbb{R}^{\left(\begin{array}{c}n+2 N \\ 2 N\end{array}\right)}$ solves the primal SDP.

(iii) If $y$ is a solution to the primal problem with $\operatorname{rank}\left(M_{N}(y)\right)=1$, then factoring $M_{N}(y)$ as column vector times row vector yields one global minimizer $z^{*}$ of the polynomial $f(x)$.

(iv) Suppose that $f_{N, \text { grad }}^{*}=f^{*}$ and $\sigma(x)=\sum_{j=1}^{m}\left(q_{j}(x)\right)^{2}$ solves the dual SDP. Then the set of all global minima of $f(x)$ equals the set of solutions $x \in \mathbb{R}^{n}$ to the following equations:

$$
\begin{aligned}
q_{j}(x) & =0, \quad j=1, \ldots, m \\
\frac{\partial f(x)}{\partial x_{i}} & =0, \quad i=1, \ldots, n .
\end{aligned}
$$


Proof Part (i) and (ii) are basically a direct application of Theorem 4.2 in [13. The hypotheses of that theorem are verified by an "epsilon argument" and applying our Theorem 3.5 Let us prove part (iii). Since the moment matrix $M_{N}(y)$ has rank one, there exists a vector $z \in \mathbb{R}^{n}$ such that $y=\operatorname{mon}_{N}(z)$. The strong duality result in (i) implies that

$$
f(z)=f^{T} y=f_{N, \text { mom }}^{*}=f_{N, \text { grad }}^{*}
$$

Since $f_{N, \text { grad }}^{*}$ is a lower bound for $f(x)$, we conclude that this lower bound is attained at the point $z$. Therefore, $f_{N, \text { grad }}^{*}=f^{*}$ and $z$ is a global minimizer. Part (iv) is straightforward.

Since all arithmetic operations are floating point operations, the numerical dual solution $y$ may have the property that the moment matrix $M_{N}(x)$ does not have rank one but is very close to a rank one matrix. In practice, we therefore need to make an approximation. Summarizing our discussion, we get the following algorithm for global minimization of polynomials.

Algorithm 4.3 Computing a global minimum of a polynomial whose infimum is obtained.

Input: A polynomial $f(x)$ of even degree $d$ in $n$ variables $x=\left(x_{1}, \ldots, x_{n}\right)$. A threshold $\varepsilon>0$.

Output: A point $z \in \mathbb{R}^{n}$ at which the polynomial $f(x)$ attains its global minimum.

Algorithm: Initialize $N=d / 2$.

Step 1 Solve pair of primal SDP and dual SDP described above.

Step 2 For the returned solution $y$ to $P_{N}$, find the rank of moment matrix $M_{N}(y)$.

Step 3 If $\operatorname{rank}\left(M_{N}(y)\right)=1$, find $z \in \mathbb{R}^{n}$ such that $y=\operatorname{mon}_{N}(z)$; return $z$, and stop.

Step 4 Find the eigenvector $u$ of $M_{N}(y)$ corresponding to the largest eigenvalue. Normalize $u$ so that $u_{1}=1$ and set $z=\left(u_{2}, \ldots, u_{n+1}\right)$.

Step 5 If $f(z)-f_{N, \text { grad }}^{*}<\left|f_{N, \text { grad }}^{*}\right| \varepsilon$, return $z$ and stop; otherwise, $N=N+1$, return to Step 1 and run it to increase the numerical accuracy of the solution $y$.

\subsection{What if the gradient ideal is not radical ?}

The lack of radicalness of the gradient ideal $\mathcal{I}_{\text {grad }}(f)$ would be an obstacle for our algorithm. First of all, this does not happen often in practice because $\mathcal{I}_{\text {grad }}(f)$ is generally radical. The following result is proved by standard arguments of algebraic geometry. We omit the proof.

Proposition 4.4 For almost all polynomials $f$ in the finite-dimensional vector space $\mathbb{R}[x]_{d}$, the gradient ideal $\mathcal{I}_{\text {grad }}(f)$ is radical and the gradient variety $V_{\text {grad }}(f)$ is a finite subset of $\mathbb{C}^{n}$.

Let us now consider the unlucky case when $\mathcal{I}_{\text {grad }}(f)$ is not radical. This happened for instance, in Example 3.4 In theory, one can replace the gradient ideal $\mathcal{I}_{\text {grad }}(f)$ by its radical $\sqrt{\mathcal{I}_{\text {grad }}(f)}$ in our SOS optimization problem. This is justified by the following result.

Corollary 4.5 If a polynomial $f(x)$ attains its infimum $f^{*}$ over $\mathbb{R}^{n}$ then $f(x)-f^{*}$ is $S O S$ modulo the radical $\sqrt{\mathcal{I}_{\text {grad }}(f)}$ of the gradient ideal.

Proof Consider the decomposition (3.2) and form the SOS polynomial $q(x)$ in (3.3). Since $f(x)-q(x)$ vanishes on the gradient variety $V\left(\mathcal{I}_{\text {grad }}(f)\right)=V\left(\sqrt{\mathcal{I}_{\text {grad }}(f)}\right)$, Hilbert's Strong Nullstellensatz implies that $f(x)-q(x) \in \sqrt{\mathcal{I}_{\text {grad }}(f)}$.

Suppose we could compute a set of polynomials $\left\{h_{1}, h_{2}, \ldots, h_{r}\right\}$ which generate the radical $\sqrt{\mathcal{I}_{\text {grad }}(f)}$ of the Jacobian ideal. Then we can replace the partial derivatives $\frac{\partial f}{\partial x_{i}}$ by the polynomials $h_{j}$ in the SOS program (4.1). The resulting SDP will always have the property that $f_{\text {grad }}^{*}=f^{*}$ provided this infimum is attainable. While there are known algorithms for computing radicals (see e.g. 9, 12]), and they are implemented in various computer algebra 
systems, running these algorithms is very time-consuming and may not terminate. We believe that replacing $\mathcal{I}_{\text {grad }}(f)$ by its radical $\sqrt{\mathcal{I}_{\text {grad }}(f)}$ is not a viable option for efficient optimization algorithms. However, it is conceivable that some polynomials in $\sqrt{\mathcal{I}_{\text {grad }}(f)} \backslash \mathcal{I}_{\text {grad }}(f)$ are known to the user (for instance, from the geometry of the problem at hand). Including such polynomials in the sum of (4.1), will surely enhance the speed of convergence of the sequence of lower bounds $f_{\text {grad }, N}^{*}, f_{\text {grad }, N+1}^{*}, \ldots \longrightarrow f^{*}$.

\section{Constrained Optimization}

This section discusses how to generalize the method in Section 4 to minimize a polynomial function subject to polynomial equality constraints. The conditions for optimality are now expressed using the KKT (Karush-Kuhn-Tucker) equations instead of the gradient ideal. We need to reformulate the problem accordingly. Similar results hold as in Section 4.

We consider the following constrained optimization problem involving polynomials in $\mathbb{R}[x]$ :

$$
\begin{aligned}
f^{*}=\min & f(x) \\
\text { s.t. } & g_{i}(x)=0, \quad i=1, \ldots, m .
\end{aligned}
$$

One lower bound can be found by SOS relaxation

$$
f_{\text {sos }}^{*}=\max _{\substack{\gamma \in \mathbb{R} \\ \phi_{i}(x) \in \mathbb{R}[x]}} \gamma \quad \text { subject to } f(x)-\gamma-\sum_{i} g_{i}(x) \phi_{i}(x) \succeq_{\text {sos }} 0 .
$$

There are several recent papers 13, 14, 21 on solving this kind of constrained problem using SOS or moment matrix techniques. The convergence of their methods is based on the assumption that the real variety $V^{\mathbb{R}}\left(g_{1}, \ldots, g_{m}\right)$ is compact or even finite, which allows the application of Putinar's Theorem 2.5 When $V^{\mathbb{R}}\left(g_{1}, \ldots, g_{m}\right)$ is compact, the methods may not converge within finitely many steps. Laurent 14 established the finite convergence of moment matrix techniques when $V^{\mathbb{R}}\left(g_{1}, \ldots, g_{m}\right)$ is finite. However, if $V^{\mathbb{R}}\left(g_{1}, \ldots, g_{m}\right)$ is not compact, then $f_{\text {sos }}^{*}$ may be smaller than $\left.f^{*}(13] 22\right)$; or even if $V^{\mathbb{R}}\left(g_{1}, \ldots, g_{m}\right)$ is compact, we may just get a sequence of bounds that converge to $f^{*}$ as the degrees of $\phi_{i}$ go to infinity [13].

As is well-known in optimization, the local or global optimal solutions to problem (5.1)- (5.2) satisfy (under some mild conditions) the KKT (Karush-Kuhn-Tucker) system of equations

$$
\begin{aligned}
\nabla f(x)+\sum_{i} \lambda_{i} \nabla g_{i} & =0 \\
g_{i}(x) & =0 .
\end{aligned}
$$

Just like the unconstrained case where we used the gradient variety, we propose to find a lower bound for $f(x)$ on the variety defined by the KKT equations. We define the KKT ideal

$$
I_{k k t}=\left\{p(x, \lambda) \in \mathbb{R}[x, \lambda]: p(x, \lambda)=\sum_{j}\left(\frac{\partial f}{\partial x_{j}}+\sum_{i} \lambda_{i} \frac{\partial g_{i}}{\partial x_{j}}\right) \eta_{j}(x, \lambda)+\sum_{i} g_{i}(x) \phi_{i}(x, \lambda)\right\} .
$$

Let $I_{\ell, k k t}$ denote the finite-dimensional $\mathbb{R}$-linear subspace of the ideal $I_{k k t}$ consisting of all polynomials which have a representation as above where each summand has degree at most $\ell$.

A lower bound for (5.1)- 5.2 can be found by solving the SOS programming problem

$$
\begin{aligned}
& f_{N, k k t}^{*}=\max _{\gamma \in \mathbb{R}, \sigma \in \mathbb{R}[x, \lambda]_{2 N}} \gamma \quad \text { subject to } \sigma(x, \lambda) \succeq_{\text {sos }} 0 \\
& \text { and } f(x)-\gamma=\sigma(x, \lambda) \bmod I_{2 N, k k t .} .
\end{aligned}
$$


Just like in Section 4, we call (5.6) - (5.7) the dual SDP formulation of our problem.

\subsection{Convergence of the lower bounds}

By Parrilo's Theorem 2.6 we have the following result:

Corollary 5.1 If the set of complex solutions to the KKT system (5.4)- 5.5) is finite and the ideal $I_{k k t}$ is radical, then $f_{N, k k t}^{*}=f^{*}$ when $N$ is large enough.

We introduce the $K K T$ variety $V_{k k t}=V\left(I_{k k t}\right)=\left\{(x, \lambda) \in \mathbb{C}^{n+m}:\right.$ [5.4 - (5.5) holds $\}$ and its subset of real points $V_{k k t}^{\mathbb{R}}=V^{\mathbb{R}}\left(I_{k k t}\right)=V_{k k t} \cap \mathbb{R}^{n+m}$. Corollary 5.1 is limited by the restrictive hypothesis that $V_{k k t}$ is a finite set. It is our objective to remove this hypothesis.

Lemma 5.2 The function $f(x)$ is constant on every irreducible subvariety $W$ of the KKT variety $V_{k k t}$ which contains a real point.

Proof The polynomials $f(x)$ and $f(x)+\sum_{i=1}^{m} \lambda_{i} g(x)$ represent the same function on $V_{k k t}$. Since $V_{k k t}$ is the gradient variety of the latter polynomial, the assertion follows from Theorem 3.3

Similarly, we can derive the following result from Theorem 3.1 and Theorem 3.5

Theorem 5.3 Suppose that either $f(x)$ is positive on $V_{k k t}$, or $f(x)$ is nonnegative on $V_{k k t}$ and $I_{k k t}$ is a radical ideal. Then $f(x)$ is a sum of squares in the residue ring $\mathbb{R}[x, \lambda] / I_{k k t}$.

¿From this we get the following convergence result for the optimization problem (5.6)-(5.7).

Corollary 5.4 Assume the optimality conditions (5.4)-(5.5) hold at all the global optima of constrained optimization [5.1)- (5.2). Then we have $\lim _{N \rightarrow \infty} f_{N, k k t}^{*}=f^{*}$. Furthermore, if the ideal $I_{k k t}$ is radical, then $f^{*}$ is attainable, i.e., there exist $S O S$ polynomial $\sigma(x, \lambda)$ such that

$$
f(x)-f^{*}=\sigma(x, \lambda) \quad \bmod I_{2 N, k k t}
$$

for some large enough integer $N$.

Corollary 5.4 says that we do not need $V^{\mathbb{R}}\left(g_{1}, \cdots, g_{m}\right)$ to be finite or compact in order for the conclusion of Corollary 5.1 to be true.

The drawback of formulation (5.6)-(5.7) is that there are new variables (Lagrange multipliers) $\lambda_{1}, \cdots, \lambda_{m}$. The computation is expensive if there are many constraints, but it is satisfactory for a few constraints. The structure must be exploited for efficient computations.

\subsection{Duality and an Algorithm}

This subsection deals with the duality of problem (5.6)-5.7 and provides an algorithm to minimize $f(x)$ under the constraints. All polynomials will lie in the polynomial ring $\mathbb{R}[x, \lambda]$.

In (5.6) - (5.7), $f(x)$ can be thought of as a polynomial in the variables $(x, \lambda)$, and the KKT system (5.4)- 5.5 is the constraint. Then (see 13 14) its primal SDP formulation is

$$
\begin{aligned}
f_{N, k \text { ktmom }}^{*}=\min _{y} & f^{T} y \\
\text { s.t. } & M_{N-e_{i}}\left(g_{i} * y\right)=0, i=1, \cdots, m \\
& M_{N-d_{k k t}}\left(\tilde{f}_{j} * y\right)=0, j=1, \cdots, n \\
& M_{N}(y) \succeq 0, y_{0}=1 .
\end{aligned}
$$

where $e_{i}=\left\lceil\operatorname{deg}\left(g_{i}\right) / 2\right\rceil, d_{k k t}=\max \left(\lceil(d-1) / 2\rceil, e_{1}, \cdots, e_{m}\right)$ and $\tilde{f}_{j}$ is the vector of coefficients of $\frac{\partial f}{\partial x_{j}}+\sum_{i} \lambda_{i} \frac{\partial g_{i}}{\partial x_{j}}$. We have the following result which is analogous to Theorem 4.2 
Theorem 5.5 Use the notations in the above. Assume $f(x)$ attains its infimum $f^{*}$ on the KKT system [5.4- 5.5). Then the following holds:

(i) $f_{N, k k t m o m}^{*}=f_{N, k k t}^{*}$ and hence $\lim _{N \rightarrow \infty} f_{N, k k t m o m}^{*}=f^{*}$, i.e., strong duality holds.

(ii) Suppose that $f_{N, k k t}^{*}=f^{*}$ for some $N$. If a feasible $x^{*}=\left(x_{1}^{*}, \cdots, x_{n}^{*}\right)^{T}$ minimizes $f(x)$ and $\lambda^{*}$ is the corresponding Lagrangian multiplier, then $y^{*}=\operatorname{mon}_{2 N}\left(\left(x^{*}, \lambda^{*}\right)\right)$ is a solution to the primal problem.

(iii) If $y$ is a solution to the primal $S D P$ such that $\operatorname{rank}\left(M_{N}(y)\right)=1$, then one point $z \in \mathbb{R}^{n}$ which minimizes $f(x)$ subject to the equality constraints can be extracted.

(iv) When $f_{N, k k t}^{*}=f^{*}$, all the optimal points $x$ minimizing $f(x)$ and their Lagrangian multipliers $\lambda$ can be obtained by solving the polynomial system

$$
\begin{aligned}
q_{j}(x, \lambda) & =0, j=1, \cdots, \ell \\
\frac{\partial f(x)}{\partial x_{j}}+\sum_{i=1}^{m} \lambda_{i} \frac{\partial g_{i}(x)}{\partial x_{j}} & =0, j=1, \cdots, n \\
g_{i}(x) & =0, i=1, \cdots, m
\end{aligned}
$$

where $\sigma(x, \lambda)=\sum_{j=1}^{\ell}\left(q_{j}(x, \lambda)\right)^{2}$ solves problem [5.6)- 5.7).

Since we perform floating point operations, the obtained numerical dual solution $y$ may not make that the moment matrix $M_{N}(x)$ have rank one, although such a solution exists by Theorem 5.5. We need to make approximations in practice. This leads to the following

Algorithm 5.6 Computing a minimum of a polynomial subject to equality constraints.

Input: Polynomials $f(x), g_{1}(x), \cdots, g_{m}(x)$, and an accuracy parameter $\varepsilon$.

Output: A solution $z \in \mathbb{R}^{n}$ to the optimization problem (5.1)- (5.2).

Algorithm: Initialize $N=2 d_{k k t}+2$.

Step 1 Solve the primal and dual SDP.

Step 2 For the returned solution $y$ to $P_{N}^{k k t}$, find the rank of moment matrix $M_{N}(y)$.

Step 3 If $\operatorname{rank}\left(M_{N}(y)\right)=1$, find the vector $z$ such that $y=\operatorname{mon}_{N}(z)$; return $z$, and stop.

Step 4 Find the eigenvector $u$ of $M_{N}(y)$ corresponding to the largest eigenvalue. Normalize $u$ so that $u(1)=1$ and set $z=u(2: n+1)$.

Step 5 If $f(z)-f_{N, k k t}^{*}<\left|f_{N, k k t}^{*}\right| \varepsilon$, return $z$ and stop; otherwise, $N=N+1$ and then go to Step 1.

Remark: Suppose that we have additional inequality constraints $h_{j}(x) \geq 0$ in the problem (5.1) - (5.2). Then we can write down the KKT system similarly, and we can try to minimize the objective $f(x)$ over the solution set of the KKT system. But now this set is no longer an algebraic variety but it is a semi-algebraic set. The convergence can be obtained by using Theorem [2.5] under some assumptions like the compactness of the solution set to the KKT system. However, the convergence analysis in Subsection 5.1 cannot be generalized directly. But the duality and algorithm in Subsection 5.2 are similar. Like the equality constraint case, this method is not practical if there are many inequality constraints. Some coordinate transformations and preprocessing are required for efficient computations. 


\section{Numerical Experiments}

The examples in this section have been computed using the software SOSTOOLS [20. In Subsection 6.1 we compare our formulation (4.1) with the formulation (1.2) by testing the family of polynomials considered in 18. ¿From the comparison tables listed below, we see that our new formulation (4.1) is faster by roughly a quarter when compared to (1.2). In Subsection 6.2, we test our method on examples where the lower bound $f_{\text {sos }}^{*}$ is strictly less than $f^{*}$. In all cases our lower bound $f_{N, \text { grad }}^{*}$ equals $f^{*}$ within rounding errors for suitable $N$.

\subsection{Testing on the Parrilo-Sturmfels family of polynomials}

In this subsection we consider the following family of polynomials of even degree $d$,

$$
f\left(x_{1}, \cdots, x_{n}\right)=x_{1}^{d}+\cdots+x_{n}^{d}+g\left(x_{1}, \cdots, x_{n}\right),
$$

where $g \in \mathbb{R}[x]$ is a random polynomial of degree $\leq d-1$ whose coefficients are uniformly distributed between $-K$ and $K$, for a fixed positive integer $K$. This family of polynomials was considered in [18] where it was shown experimentally that the SOS formulation (1.2) almost always yields the global minimum. Without loss of generality, we can set $K=1$, because any $f(x)$ in the above form can be scaled to have coefficients between -1 and 1 by taking

$$
f_{s}\left(x_{1}, \cdots, x_{n}\right)=\alpha^{-d} \cdot f\left(\alpha x_{1}, \cdots, \alpha x_{n}\right)
$$

for some properly chosen $\alpha$. As observed in [18, this scaling will greatly increase the stability and speed of the numerical computations involved in solving the primal-dual SDP.

We ran a large number of randomly generated examples for various values of $d$ and $n$. The comparison results are in listed in Table 1 and Table 4 The computations were performed on a Dell Laptop with a Pentium IV 2.0 GHz and 512MB of memory. Table 1 is the comparison of the lower bounds by formulation (1.2) and (4.1). Taking $N=d / 2$ in Algorithm 4.3] appears to be good enough in practice for minimizing the Parrilo-Sturmfels polynomials. Our experiments show that increasing $N$ above $d / 2$ will not increase the lower bound significantly.

From Table 1 we can see that the lower bounds $f_{\text {sos }}^{*}$ and $f_{N, \text { grad }}^{*}$ are close, agreeing to their leading 8 to 10 decimal digits, which confirms the observation made in 18 that almost all the polynomials gotten by subtracting their infima are SOS. Tables 24 are comparisons of running time in CPU seconds for formulations (1.2) and 4.1. The symbol "-" in the tables means that the computation takes more than one hour and we then terminate it. And "**" means we use a different scaling as described below.

Our formulation 4.1] uses about three quarters of the running time used by formulation (1.2). This may be unexpected since the use of gradients introduces many new variables. While we are not sure of the reason, one possible explanation is that adding gradients improves the conditioning and makes the interior-point algorithm for solving the SDP converge faster.

The numerical performance is subtle in this family of test polynomials. In the cases $(n, d)=$ $(4,10)$ or $(n, d)=(5,10)$, our formulation 4.1) has numerical trouble, while (1.2) does not, and yet (4.1) is still faster than (1.2). However, for these two cases, if we scale $f\left(x_{1}, \ldots, x_{n}\right)$ so that the coefficients of $g\left(x_{1}, \ldots, x_{n}\right)$ belong to $[-0.1,0.1]$, both 1.2 and 4.1] do not have numerical trouble, and formulation (4.1) is still faster than (1.2). In Table 4 we see that the time ratio between (4.1) and (1.2) under this scaling is smaller than the time ratio for other values of $(n, d)$. So numerical comparisons in Tables 14 for $(n, d)=(4,10)$ or $(n, d)=(5,10)$ are implemented under this new scaling, while for other values of $(n, d)$ we still use the old scaling where the coefficients of $g\left(x_{1}, \ldots, x_{n}\right)$ belong to $[-1,1]$. A stability analysis for the scaling and the speed-up caused by adding gradients may be a future research topic. 
"-" means the computation is terminated if it takes more than one hour; "*" means the coefficients of $g\left(x_{1}, \cdots, x_{n}\right)$ are scaled to belong to $[-0.1,0.1]$.

\begin{tabular}{c|rrrrrrrr}
$d \backslash n$ & 3 & 4 & 5 & 6 & 7 & 8 & 9 & 10 \\
\hline 4 & 5 & 7 & 9 & 10 & 11 & 13 & 14 & 15 \\
6 & 10 & 19 & 38 & 41 & 232 & - & - & - \\
8 & 17 & 78 & 186 & 233 & - & - & - & - \\
10 & 40 & $39^{*}$ & $102^{*}$ & - & - & - & - & -
\end{tabular}

Table 1: The relative difference $\frac{\left|f_{N, \text { grad }}^{*}-f_{\text {sos }}^{*}\right|}{\left|f_{\text {sos }}^{*}\right|} \times 10^{10}$, with $N=d / 2$.

\begin{tabular}{c|rrrrrrrr}
$d \backslash n$ & 3 & 4 & 5 & 6 & 7 & 8 & 9 & 10 \\
\hline 4 & 0.16 & 0.24 & 0.42 & 0.86 & 1.86 & 7.56 & 25.85 & 73.69 \\
6 & 0.32 & 1.17 & 8.40 & 49.04 & 309.66 & - & - & - \\
8 & 1.10 & 12.23 & 173.98 & 1618.86 & - & - & - & - \\
10 & 3.15 & $64.48^{*}$ & $2144.04^{*}$ & - & - & - & - & -
\end{tabular}

Table 2: Running time in CPU seconds via traditional SOS approach (1.2)

\begin{tabular}{c|rrrrrrrr}
$d \backslash n$ & 3 & 4 & 5 & 6 & 7 & 8 & 9 & 10 \\
\hline 4 & 0.12 & 0.18 & 0.32 & 0.68 & 1.46 & 5.65 & 18.85 & 54.97 \\
6 & 0.23 & 0.91 & 6.39 & 35.16 & 241.71 & - & - & - \\
8 & 0.84 & 9.54 & 129.53 & 1240.23 & - & - & - & - \\
10 & 2.59 & $45.14^{*}$ & $1539.80^{*}$ & - & - & - & - & -
\end{tabular}

Table 3: Running time in CPU seconds via our approach 4.1), with $N=d / 2$.

\begin{tabular}{c|rrrrrrrr}
$d \backslash n$ & 3 & 4 & 5 & 6 & 7 & 8 & 9 & 10 \\
\hline 4 & 0.75 & 0.75 & 0.76 & 0.79 & 0.78 & 0.74 & 0.73 & 0.75 \\
6 & 0.72 & 0.77 & 0.76 & 0.72 & 0.78 & - & - & - \\
8 & 0.76 & 0.78 & 0.74 & 0.76 & - & - & - & - \\
10 & 0.82 & $0.70^{*}$ & $0.71^{*}$ & - & - & - & - & -
\end{tabular}

Table 4: The ratio of CPU seconds between (1.2) and (4.1), with $N=d / 2$. 


\subsection{Other Examples}

The following examples demonstrate the effectiveness of our Algorithm 4.3 for a sample of polynomials that have been discussed in the SOS optimization literature.

Homogeneous Polynomials Let $f(x)$ be a homogeneous polynomial. Regardless of whether $f(x)$ is non-negative, we always have $f_{N, \operatorname{grad}}^{*}=0$ for any $N \geq d / 2$. This comes from the identity $f(x)=\frac{1}{d} \cdot \sum_{i} x_{i} \frac{\partial f}{\partial x_{i}}$, which implies that $f(x)$ lies in its gradient ideal $\mathcal{I}_{\text {grad }}(f)$. In order to test global non-negativity of a homogeneous polynomial $f(x)$, we can apply Algorithm 4.3 to a dehomogenization of $f(x)$, as shown in Examples 2 and 3 below.

Example 1: $f(x, y)=x^{2} y^{2}\left(x^{2}+y^{2}-1\right)$. This polynomial is taken from [13]. It has global minimum value $f^{*}=-1 / 27=-0.03703703703703 \ldots$ However, $f_{\text {sos }}^{*}=-33.157325$ is considerably smaller than $f^{*}$. If we minimize $f(x)$ over its gradient ideal with $N=4$, then we get $f_{4, \text { grad }}^{*}=-0.03703703706212$. The difference equals $f^{*}-f_{4, \text { grad }}^{*} \approx 2.50 \cdot 10^{-11}$.

Example 2: The polynomial $f(x, y)=x^{4} y^{2}+x^{2} y^{4}+1-3 x^{2} y^{2}$ is obtained from the Motzkin polynomial by substituting $z=1$ as in [19]. We have $f^{*}=0>f_{\text {sos }}^{*}=-\infty$. However, if we minimize $f(x, y)$ over its gradient ideal with $N=4$, we get $f_{4, \text { grad }}^{*}=-6.1463 \cdot 10^{-10}$.

Example 3: The polynomial $f(x, y)=x^{4}+x^{2}+z^{6}-3 x^{2} z^{2}$ is obtained from the Motzkin polynomial by substituting $y=1$. Now, $f^{*}=0>f_{\text {sos }}^{*}=-729 / 4096$. However, if we minimize $f(x, z)$ over its gradient ideal with $N=4$, we get $f_{4, \text { grad }}^{*}=-9.5415 \cdot 10^{-12}$.

Example 4: Consider the following constrained optimization problem:

$$
\min _{x, y, z} x^{2} y^{2}\left(x^{2}+y^{2}+z^{2}-1\right) \quad \text { subject to } z=0 .
$$

This one is modified from Example 1. The exact lower bound is still $-1 / 27$. However, we can not find a general polynomial $\phi(x)$ and SOS polynomial $\sigma(x, y, z)$ such that

$$
x^{2} y^{2}\left(x^{2}+y^{2}+z^{2}-1\right)+1 / 27=\sigma(x, y, z)+\phi(x, y, z) z .
$$

To see why, plug $z=0$ in the above identity getting $x^{2} y^{2}\left(x^{2}+y^{2}-1\right)+1 / 27$, which can not be SOS as we see in Example 1. Now we solve this problem using formulation (5.6) - (5.7) with $N=4$. The lower bound we get is $-.03703704=-1 / 27-3.9690 \cdot 10^{-9}$.

\section{Conclusions}

This paper proposes a method for minimizing a multivariate polynomial $f(x)$ over its gradient variety. We assume that the infimum $f^{*}$ is attained. Every polynomial which is strictly positive on its real gradient variety is SOS modulo its gradient ideal, even if the gradient variety is not zero-dimensional or radical. This fact implies that we can find a sequence of lower bounds $\left\{f_{N, \text { grad }}^{*}\right\}$ which converges to $f^{*}$. Moreover, if the gradient ideal is radical, we showed that every nonnegative polynomial is also SOS modulo its gradient ideal, which implies that $f_{N, \text { grad }}^{*}=f^{*}$ for some integer $N$. This finite convergence property holds for random polynomials by Proposition 4.4 Our method can also be generalized to constrained polynomial optimization. Instead of using gradients, we minimizing the objective polynomial over the variety defined by its KKT system. Similar results hold as in the unconstrained case.

Numerical experiments with SOSTOOLS suggest that our algorithm is effective for unconstrained polynomial optimization. Our method (4.1) with gradients is faster than the method (1.2) without gradients. Our method is also very good for equality constrained optimization, when the number of equality constraints are small compared with the number of decision 
variables. When there are many equality or inequality constraints, the structure of the KKT system must be exploited for computation efficiency. This may be a future research topic.

Acknowledgments. The authors thank Claus Scheiderer for very helpful comments and for providing the example at the end of Section 2. Jean Lasserre and Pablo Parrilo suggested one example in Section 3. We are grateful to Monique Laurent for reviewing an early manuscript version and fruitful suggestions that helped improved this paper. Jiawang Nie was supported in part by National Science Foundation (ELA-0122599). Bernd Sturmfels was supported in part by the National Science Foundation (DMS-0200729).

\section{References}

[1] C. Berg. The multidimensional moment problem and semi-groups, in Moments in Mathematics, H.J. Landau, ed., AMS, Providence, RI, 1980, pp. 110-124.

[2] J. Bochnak, M. Coste and M-F. Roy. Real Algebraic Geometry, Springer, 1998.

[3] G. Blekherman. Volumes of nonnegative polynomials, sums of squares, and powers of linear forms, preprint, arXiv:math.AG/0402158

[4] E. Becker and R. Neuhaus: Computation of real radicals of polynomial ideals. Computational algebraic geometry (Nice, 1992), 1-20, Progress in Mathematics, 109, Birkhäuser, Boston, MA, 1993.

[5] E. Becker and R. Neuhaus: Computation of real radicals of polynomial ideals. II. J. Pure Appl. Algebra 124 (1998) 261-280.

[6] D.A. Cox, J.B. Little, and D.O'Shea. Ideals, Varieties and Algorithms: an Introduction to Computational Algebraic Geometry and Commutative Algebra, Second Edition. Undergraduate Texts in Mathematics. Springer-Verlag, New York, 1997.

[7] D.A. Cox, J.B. Little, and D.O'Shea. Using Algebraic Geometry, Graduate Texts in Mathematics, Vol. 185. Springer-Verlag, New York, 1998.

[8] D. Eisenbud, Commutative Algebra with a View Toward Algebraic Geometry, Graduate Texts in Mathematics, Vol. 150. Springer-Verlag, New York, 1995.

[9] E. Fortuna, P. Gianni, and B. Trager: Derivations and radicals of polynomial ideals over fields of arbitrary characteristic. Computer algebra (London, ON, 2001). J. Symbolic Comput. 33 (2002), no. 5, 609-625.

[10] B. Hanzon and D. Jibetean: Global minimization of a multivariate polynomial using matrix methods. Journal of Global Optimization, 27:1-23, 2003

[11] D. Jibetean and M. Laurent. Converging SDP bounds for global unconstrained polynomial optimization. Preprint, 2004. Website: www.cwi.nl/ monique.

[12] T. Krick and A. Logar: An algorithm for the computation of the radical of an ideal in the ring of polynomials. Applied algebra, algebraic algorithms and error-correcting codes (New Orleans, LA, 1991), 195-205, Lecture Notes in Comput. Sci., 539, Springer, Berlin, 1991.

[13] J. Lasserre. Global optimization with polynomials and the problem of moments. SIAM J. Optim., 11 (2001), No. 3, 796-817.

[14] M. Laurent. Semidefinite representations for finite varieties. Preprint, 2004. Website: www.cwi.nl/ monique. 
[15] Y. Nesterov. Squared functional systems and optimization problems. High Performance Optimization (H.Frenk et al., eds), Kluwer Academic Publishers, 2000, pp.405-440.

[16] J. Nie and J. W. Demmel. Minimum ellipsoid bounds for solutions of polynomial systems via sum of squares, to appear in Journal of Global Optimization, arXiv:math.0C/0411122

[17] P. Parrilo. Structured Semidefinite Programs and Semialgebraic Geometry Methods in Robustness and Optimization, Ph.D Thesis, California Institute of Technology, 2000.

[18] P. Parrilo and B. Sturmfels. Minimizing polynomial functions, Proceedings of the DIMACS Workshop on Algorithmic and Quantitative Aspects of Real Algebraic Geometry in Mathematics and Computer Science (March 2001), (eds. S. Basu and L. GonzalezVega), American Mathematical Society, 2003, pp. 83-100.

[19] P. Parrilo. Semidefinite Programming relaxations for semialgebraic problems. Mathematical Programming, Ser. B 96 (2003), No. 2, 293-320.

[20] S. Prajna, A. Papachristodoulou and P. Parrilo. SOSTOOLS User's Guide. http://control.ee.ethz.ch/ parrilo/SOSTOOLS/.

[21] P. Parrilo. An explicit construction of distinguished representations of polynomials nonnegative over finite sets, IfA Technical Report AUT02-02, March 2002.

[22] M. Putinar. Positive polynomials on compact semi-algebraic sets, Ind. Univ. Math. J. 42 (1993) 203-206.

[23] B. Reznick. Some concrete aspects of Hilbert's $17^{\text {th }}$ problem. In Contemporary Mathematics, volume 253, pages 251-272. American Mathematical Society, 2000.

[24] L. Vandenberghe and S. Boyd. Semidefinite Programming, SIAM Review 38 (1996) 49-95. 